\title{
Modelling sublimation and atomic layer epitaxy in the presence of competing surface reconstructions
}

\author{
M. Ahr: 1 , M. Biehl ${ }^{1,2}$ \\ ${ }^{1}$ Institut für Theoretische Physik und Astrophysik, Julius-Maximilians-Universität Würzburg, Am Hubland, 97074 Würzburg, \\ Germany \\ 2 Sonderforschungsbereich 410, Universität Würzburg
}

(October 25, 2018)

\begin{abstract}
We present a solid-on-solid model of a binary $A B$ compound, where atoms of type $A$ in the topmost layer interact via anisotropic interactions different from those inside the bulk. Depending on temperature and particle flux, this model displays surface reconstructions similar to those of (001) surfaces of II-VI semiconductors. We show, that our model qualitatively reproduces many of the characteristic features of these materials which have been observed during sublimation and atomic layer epitaxy. We predict some previously unknown effects which might be observed experimentally. Keywords: Monte Carlo simulations, Surface relaxation and reconstruction, Models of nonequilibrium phenomena, Evaporation and sublimation, Growth
\end{abstract}

Apart from a few exceptions (eg. [1]), theoretical models of crystal surfaces in nonequilibrium situations have not taken into account the effects of surface reconstructions, so far. Consequently, a theoretical understanding of the interplay of this phenomenon with the dynamics of growing or sublimating surfaces has not yet been achieved. In this paper we will address this important question in the context of a compound material that displays a competition of different vacancy structures in the terminating layer.

Within the last years, there have been intensive experimental studies concerning the surfaces of II-VI semiconductors, see eg. [2, 3] for an overview. These studies have revealed the existence of a fairly small number of surface reconstructions, which makes them promising candidates for a theoretical modelling. In the following, we will refer mostly to experimental results for $\mathrm{CdTe}(001)$ [2-9]. Under vacuum conditions, the $\mathrm{CdTe}(001)$ surface is metal terminated. The $\mathrm{Cd}$ atoms arrange in a vacancy structure, where half of the potential $\mathrm{Cd}$ sites are empty. At low temperatures, a $c(2 \times 2)$ reconstruction is observed, in which $\mathrm{Cd}$ atoms arrange in a checkerboard like configuration. Often a contribution of $(2 \times 1)$ arrangement in rows along the (110) direction is observed. At a temperature of $\approx 300^{\circ} \mathrm{C}$, a reordering occurs, above which a $(2 \times 1)$ ordering dominates on a sublimating surface. An additional $\mathrm{Cd}$ flux stabilizes the $c(2 \times 2)$ ordering even at high temperatures. Under a Te flux, the surface is Te-terminated, with a $(2 \times 1)$ reconstruction. At small Te fluxes, the Te-coverage is 1 , at low temperatures and high Te fluxes a higher coverage of 1.5 is observed [3, 5, 6. 86]. In this Letter, we present a 2+1-dimensional solid-on-solid model of a binary compound material to get insight into the following problems: (1) Is the claim [6] of an effective thermal equilibrium of the surface layer justified under the non-equilibrium conditions of sublimation? (2) What is the effect of an external particle flux on the surface reconstruction? (3) How do reconstructions influence techniques of crystal growth like atomic layer epitaxy (ALE)?

In the terminating layer of a $\mathrm{CdTe}(001)$ surface, the potential $\mathrm{Cd}$ sites form a regular square lattice, where the simultaneous occupation of nearest neighbour $(\mathrm{NN})$ sites in the [010]-direction (denoted as y in the following) is forbidden by electron counting rules [10]. In our model, there is an attractive interaction between nearest neighbours in [110](x)-direction (coupling $\varepsilon_{x}$ ) and diagonal neighbours (coupling $\varepsilon_{d}$ ). Measuring energy in units of $\left|\varepsilon_{d}\right|$, we set $\varepsilon_{d}=-1$. A model, where the underlying crystal was assumed to be fixed, has been studied elsewhere [11]. If the energy difference between a perfect $c(2 \times 2)$ arrangement and a perfect $(2 \times 1)$ arrangement is small and positive $\left(-2<\varepsilon_{x} \leq-1.9\right)$, this model explains the $c(2 \times 2)-(2 \times 1)$ reordering as an accompanying effect of an order-disorder transition in thermal equilibrium. At low temperature $T$, the system is in an ordered phase, where $c(2 \times 2)$ dominates, above the transition it is in a globally disordered phase, where the $(2 \times 1)$ rows dominate the local environment of a particle.

In this paper, we extend this planar lattice gas to a model of a three-dimensional crystal. Currently no values of diffusion energy barriers from ab initio or semi-empirical calculations are available, and the existing experimental data are insufficient for a parameter fit. Therefore, a realistic modelling of CdTe is beyond the scope of this work, which aims at an understanding of fundamental properties of nonequilibrium crystal surfaces. Instead, we consider a model of a compound " $A B$ " with a cubic lattice and a comparatively simple potential energy surface which, although not being quantitative, reproduces essential features observed in experiments on $\operatorname{CdTe}(001)$. Here, the $A(B)$ atoms correspond to $\mathrm{Cd}(\mathrm{Te})$. Within the framework of a solid-on-solid model, we describe the crystal by a two-dimensional array $\left\{h_{x y}\right\}_{x, y=1}^{N}$ of integers which denote the height of a column of atoms. We use periodic boundary conditions. 
The interactions between the particles are described by phenomenological parameters which are supposed to include the effects of surface strain and other interactions. To model the layered structure of the CdTe crystal, we assign the odd heights to $A$ particles, and the even heights to $B$ particles. Inside the bulk of the crystal, there is an attractive interaction between the particles and their NNs which is isotropic in directions parallel to the surface. While there is no difference in the interaction of $B$ particles between the bulk and the surface, $A$ particles on the surface interact with the anisotropic interactions $\varepsilon_{x}$ and $\varepsilon_{d}$. The other parameters of the model are defined in Figure 11. Thus, the Hamiltonian of the crystal is

$$
H=\varepsilon_{A B} n_{A B}+\varepsilon_{B B} n_{B B}+\varepsilon_{b} n_{A A}^{b}+\varepsilon_{h} n_{A A}^{h}+\varepsilon_{d} n_{A A}^{d}+\varepsilon_{x} n_{A A}^{x}
$$

Here, $n_{A B}$ is the number of $A B$ bonds between the layers, $n_{B B}$ is the number of NN pairs of $B$ atoms, $n_{A A}^{b}$ the number of $A$ NN pairs inside the bulk, $n_{A A}^{h}$ the number of surface $A$ atoms next to a higher column, $n_{A A}^{d}\left(n_{A A}^{x}\right)$ the number of bindings of surface $A$ atoms to diagonal neighbours (NNs in x-direction) at the same height. The stay of particles in the wrong sublattice and the formation of NN pairs of surface $A$ atoms in y-direction is forbidden. We allow diffusion both to nearest neighbour sites and to diagonal neighbours. Additionally, we permit the diffusion of an $A B$ pair with the $B$ atom on top of the $A$ atom, if the diffusion of the $B$ atom alone would end in the wrong sublattice. This process is required to preserve the ergodicity of the system. We simulate a Kawasaki dynamics, i.e. the rate of diffusion events is $\nu_{0} \exp \left(-\left(B_{0}+\Delta H\right) / T\right)$ if $\Delta H>0$ and $\nu_{0} \exp \left(-B_{0} / T\right)$ else. The desorption of an $A(B)$ particle requires an additional activation energy $E_{A}\left(E_{B}\right)$. This is done using rejection free Monte-Carlo techniques employing a binary search tree [12]. Particles from an external source arrive at each lattice site with equal probability. If the adsorption of the particle at this site would lead to a forbidden state, it is destroyed.

This dynamics does not depend on the parameter $\varepsilon_{A B}$, so it needs not to be specified, and $\varepsilon_{B B}$ and $\varepsilon_{b}$ enter only via the sum $\varepsilon^{\prime}:=\varepsilon_{B B}+\varepsilon_{b}$. The constant prefactor $1 / t_{0}:=\nu_{0} \exp \left(-B_{0} / T\right)$ sets the timescale of the model and needs not to be specified, if we measure time in units of $t_{0}$.

In the following, we will use the parameter set $\varepsilon^{\prime}=\varepsilon_{d}=-1, \varepsilon_{h}=0, \varepsilon_{x}=-1.97, E_{A}=E_{B}=1.5$ (in units of $\left|\varepsilon_{d}\right|$ ). The fact that the difference in the surface energies between perfectly $c(2 \times 2)$ and $(2 \times 1)$ reconstructed surfaces is small is consistent with DFT calculations [7]. However, precise values of binding energies as a quantitative input to our model are not available. We have verified, that the behaviour of our model remains qualitatively the same for a wide range of parameters, as long as the basic property of a small energy difference between the reconstructions is preserved.

To characterize the surface reconstruction quantitatively, we measure the $A$-coverage $\theta_{A}=n_{A}^{\text {surf. }} / N^{2}$, and the normalized correlations $C_{A A}^{d}:=n_{A A}^{d} /\left(4 n_{A}^{\text {surf. }}\right)$ and $C_{A A}^{x}:=n_{A A}^{x} /\left(2 n_{A}^{\text {surf. }}\right)$, which quantify the fraction of $A$-atoms on the surface which are incorporated in locally $c(2 \times 2)$ or $(2 \times 1)$ reconstructed areas, respectively. Additionally, we calculate LEED intensities to model diffraction experiments. Neglecting multiple scattering, this is done by calculating the fourier transform of the array $\left\{\exp \left(i \Delta k h_{x y}\right)\right\}_{x, y=1}^{N}$, where equal diffraction intensities for $A$ and $B$ atoms have been assumed. The antiphase condition between different layers is $\Delta k=\pi / 2$.

Figure 22a shows the evolution of an initially $B$-terminated, flat surface under vacuum at a temperature $T=0.5$. Initially, $\theta_{A}$ increases from zero to an asymptotic value close to 0.5 . We find, that this increase can be fitted with an exponential relaxation, $\theta_{A}=\theta_{A}^{\infty}(1-\exp (-t / \tau))$. Here, $\tau$ is the time constant of the decay of the $B$-terminated surface. We find, that the temperature dependence of the corresponding desorption probability $p \propto 1 / \tau$ follows an Arrhenius law, $p \propto \exp \left(-E_{a c t}\right.$. $\left./ T\right)$, with an activation energy $E_{a c t .}=B_{0}+3.0 \pm 0.1$ After this onset, $A$ and $B$ evaporate stoichiometrically, and sublimation proceeds in layer-by-layer mode, which reflects in the oszillations of the specular LEED intensity $I_{\text {spec }}$ in antiphase (dotted curve in Figure 2a). The dependence of the sublimation rate on $T$ follows an Arrhenius law with a higher activation energy $B_{0}+6.0 \pm 0.2$. Thus, our model reproduces the observation of Cdterminated CdTe surfaces under vacuum. Studies of surface lifetimes [5] and QMS measurements of evaporation rates [4] have also revealed significantly smaller activation energies for the decay of the Te-terminated surface $(\approx 0.7 \mathrm{eV})$ than for congruent evaporation (1.94eV in layer-by-layer mode). We note however, that in our model the microscopical energy barriers for desorption of $A$ and $B$ are equal. Therefore the difference in the macroscopic activation energies is solely due to the stabilizing effect of the surface reconstruction.

Surprisingly, $C_{A A}^{d}$ and $C_{A A}^{x}$ oszillate during sublimation, which proceeds layer by layer. Each time a complete layer has desorbed and the surface is atomically flat (maxima of $I_{\text {spec }}$ ) the $c(2 \times 2)$ reconstructed fraction of the surface is maximal. On the contrary, a rough surface with a large number of islands (minimal $I_{\text {spec }}$ ) seems to prefer the $(2 \times 1)$ reconstruction. This can be understood from the fact that the attractive lattice gas interactions are present only between particles in the same layer. Thus, the island edges impose open boundary conditions to the lattice gas of $A$ atoms on the island. In contrast to a $c(2 \times 2)$ reconstructed domain, a $(2 \times 1)$ terminated island can reduce the energy of its boundary by elongating in x-direction. Since the ground state energies of both structures are nearly degenerate, the formation of $(2 \times 1)$ may reduce the surface free energy. This picture is confirmed by the fact that islands on sublimating surfaces are indeed elongated (See Figure [a,b) [13]. 
Experiments have revealed a high density of steps on CdTe surfaces due to the intrinsic surface morphology [ 4 , which leads to a dominant contribution of step flow sublimation. To incorporate this effect in our model, we have performed simulations of vicinal surfaces [13]. We find, that the oszillations in the correlations dissappear for terrace widths smaller than $\approx 50$ lattice constants, where step flow sublimation dominates. Then, the correlations become stationary apart from statistical fluctuations. Figure 3 a shows the $T$-dependence of $\theta_{A}, C_{A A}^{d}, C_{A A}^{x}$ and the sublimation rate $r_{\text {sub }}$ at a step distance of $32 \sqrt{2}$ lattice constants. The steps were oriented at an angle of $45^{\circ}$ to the x-axis, which corresponds to the preferential (100)-orientation of steps observed on $\mathrm{CdTe}(001)$ [9]. Snapshots of the surfaces are shown in Figure 4. Clearly, at $T=0.55$ there is a transition from a $c(2 \times 2)$ configuration at low temperature to a high-temperature regime where the $(2 \times 1)$ ordering dominates and the material is sublimating. This is the conterpart of the phase transition observed in our two-dimensional lattice gas model. In the investigated temperature range $\theta_{A}$ decreases only slightly from $\theta_{A}=0.498$ at $T=0.4$ to $\theta_{A}=0.41$ at $T=0.8$.

To get insight into the behaviour of a material in an MBE environment, it is important to understand how the structure of the surface changes when it is exposed to a particle beam. To this end, we have simulated surfaces which were exposed to a flux of pure $A$ or $B$. Figure $3 \mathrm{~b}$,c shows the quantities $\theta_{A}, C_{A A}^{d}$ and $C_{A A}^{x}$ as functions of the adsorption rate at a temperature $T=0.4$, which is far below the $c(2 \times 2)-(2 \times 1)$ reordering under vacuum, and at $T=0.57$, which is slightly above the transition. Applying $B$ fluxes, we can regulate $\theta_{A}$ to values smaller than those close to 0.5 observed under vacuum. We find, that a decrease of $\theta_{A}$ leads to a significant increase in $C_{A A}^{x}$ (Fig. $3 \mathrm{~b}, \mathrm{c}$ ). This effect is analogous to the higher fraction of $(2 \times 1)$ ordering on surfaces with a large number of islands: at low $\theta_{A}, A$-covered islands form on the surface, whose boundaries favour the arrangement of $A$ atoms in rows. On the contrary, an $A$ flux increases $\theta_{A}$ and $C_{A A}^{d}$ and leads to the formation of a dominant $c(2 \times 2)$ reconstruction even at temperatures above the reordering under vacuum. The reappearance of a $c(2 \times 2)$ reconstruction under a $\mathrm{Cd}-\mathrm{flux}$ at high temperature and the formation of Te-terminated surfaces with coverage 1 even at low Te fluxes are well known from experiments on CdTe [3]. In these experiments however, the formation of Te dimers on the surface was observed, an effect which is not present in our simple model assuming isotropic interactions between $B$ atoms.

It has been argued [6], that the CdTe surface is close to thermal equilibrium during sublimation, since the bulk serves as a particle reservoir which defines chemical potentials for both elements. Comparing our solid-on-solid model with the two-dimensional anisotropic lattice gas, we calculate the equilibrium values of $C_{A A}^{d}, C_{A A}^{x}$ in the planar lattice gas model at the value of $\theta_{A}$ we measure for $A B$ at a given temperature, which can be done using transfer matrix techniques [11]. Although the non-equilibrium conditions of sublimation enhance the dominance of $C_{A A}^{x}$ over $C_{A A}^{d}$ in the high temperature regime, we find qualitative agreement in the behaviour of $C_{A A}^{d}$ and $C_{A A}^{x}$. However, a simple mapping of the surface layer of the sublimating system on a 2D lattice gas in thermal equilibrium is not possible.

Atomic layer epitaxy (ALE) provides a scenario for the investigation of our model of the II-VI semiconductor surface in a situation present in technical applications. The idea is to obtain self-regulated growth by alternate deposition of pure $A$ and $B$. In the absence of reconstructions, one would expect the formation of complete monolayers of $A$ and $B$ during deposition of the elements, yielding growth at a speed of one monolayer (ML) per cycle. However, this does not necessarily apply in the presence of surfaces terminated by vacancy structures with submonolayer coverage. Experimentally, in CdTe one finds growth rates of $\approx 1 \mathrm{ML} /$ cycle only at $T<260^{\circ} C$, and growth at a speed of $\approx 0.5$ ML/cycle at higher temperatures [8, [3]. Figure 2b shows the evolution of $\theta_{A}, C_{A A}^{d}$ and $C_{A A}^{x}$ during ALE at $T=0.3$. Each cycle consists of two phases of length $t_{c y c l}=4 \cdot 10^{3} t_{0}$. In the first phase, an $A$ flux of $5 \cdot 10^{-3} \mathrm{ML} / t_{0}$ is applied, in the second phase $B$ is deposited at the same rate. Growth was started from a $B$ terminated flat surface. During the first $A$ phase the surface becomes $A$-terminated with $\theta_{A} \approx 1 / 2$. Since $T$ is far below the transition, the reconstruction is $c(2 \times 2)$. At the onset of the $B$-phase, $\theta_{A}$ decreases rapidly. This leads to an increase of $C_{A A}^{x}$, as reported above for surfaces under a small stationary $B$-flux. At the end of the cycle, $\approx 50 \%$ of the surface are covered with $B$-terminated islands, since only half of the $A$ atoms needed for a closed monolayer is present. The following $A$-phase deposits half a monolayer of $A$ again. However, now the reconstruction is preferentially $(2 \times 1)$. This is analogous to the maximum of $C_{A A}^{x}$ we observe in layer-by-layer sublimation after half a monolayer has desorbed, which is due to the influence of the island edges on the reconstruction. During the following $B$ phase, $A$ atoms and $A B$ pairs on top of the islands diffuse into the gaps between the islands, which leads to the formation of a closed $B$-terminated monolayer. In our simulations, we observe 2-3 repetitions of this cycle 13]. There are two effects, which keep it from repeating infinitely. First, we obtain a growth rate of $0.44 \mathrm{ML} /$ cycle, which is smaller than the ideal value of $0.5 \mathrm{ML} / \mathrm{cycle}$. Second, the diffusion of particles from the islands into the gaps is not complete. Both effects hinder a perfect closure of the monolayer at the end of the even cycles, which leads to a damping of the characteristic behaviour. These observations closely resemble the ideas developed to explain CdTe growth at a speed of $\approx 0.5 \mathrm{ML} /$ cycle in $[8]$.

The experimental observation of growth rates of $1 \mathrm{ML} /$ cycle in ALE at low temperatures is inconsistent with our model in its present form, since the hardcore repulsion between the $A$ atoms hinders the deposition of more than 0.5 ML of $A$. However, this effect might be explained with the existence of weakly bound precursor states, where particles could stay close to the surface until the deposition of the opposite species allows for an incorporation into the crystal. 
At higher temperatures this reservoir would be inactive due to the weakness of the binding. Future research might also address the influence of an interlayer diffusion barrier on ALE and MBE in our model. Additionally, one further step towards a more realistic modelling of CdTe should be the simulation of a zinc-blende lattice which, however, requires the specification of a greater number of parameters.

In summary, we have presented a simple model of a binary compound material which reproduces many effects observed in experiments on $\mathrm{CdTe}(001)$ qualitatively. We conclude suggesting some experiments to test our model. (1) The preparation of $\mathrm{CdTe}(001)$ surfaces with an extremely low intrinsic step density might allow for the observation of oscillations in the correlations during layer-by-layer sublimation. (2) The deposition of small amounts of Te on a $\mathrm{CdTe}(001)$ surface should lead to a preferential arrangement of the remaining Cd atoms in a $(2 \times 1)$ ordering. (3) During Cd deposition in ALE of CdTe a $c(2 \times 2)$ reconstruction is expected in cycles starting from flat Te-terminated surfaces, while in cycles starting from rough surfaces $(2 \times 1)$ should be present.

We thank W. Kinzel and M. Sokolowski for stimulating discussions and a critical reading of the manuscript. M.A. was supported by the Deutsche Forschungsgemeinschaft.

* Corresponding author. Address: Institut für Theoretische Physik und Astrophysik, Julius-Maximilians-Universität Würzburg, Am Hubland, 97074 Würzburg, Germany.

Phone: +49 (0)931 8884908

Fax: +49(0)931 8885141

E-mail: ahr@physik.uni-wuerzburg.de

[1] M. Itoh, G.R. Bell, A.R. Avery, T.S. Jones, B.A. Joyce, D.D. Vvedensky, Phys. Rev. Lett. 81(3) 633 (1998)

[2] Proceedings of the 9th International Conference on II-VI Compounds, Kyoto 1999, to be published in Journal of Crystal Growth (2000)

[3] J. Cibert, S. Tatarenko. Defect and Diffusion Forum Vols. 150-151, 1 (1997) and references therein

[4] H. Neureiter, S. Schinzer, W. Kinzel, S. Tatarenko, M. Sokolowski. Phys. Rev. B 61 (8), 5408 (2000)

[5] S. Tatarenko, B. Daudin, D. Brun, V. H. Etgens, M B. Veron. Phys. Rev. B 50 (24), 18479 (1994)

[6] H. Neureiter, S. Tatarenko, S. Spranger, M. Sokolowski. Phys. Rev. B. 62 (4), 2542, (2000)

[7] S. Gundel, A. Fleszar, W. Faschinger, W. Hanke. Phys. Rev. B 59 (23), 15261 (1999)

[8] B. Daudin, D. Brun-Le Cunff, S. Tatarenko. Surf. Sci. 352-354, 99 (1996) and B. Daudin, S. Tatarenko, D. Brun-Le Cunff, Phys. Rev. B 52 (11), 7822, 1995

[9] D. Matrou, J. Eymery, P. Gentile, N. Magnea. J. Cryst. Growth 184/185, 203 (1998) and D. Martrou, J. Eymery, N. Magnea, Phys. Rev. Lett. 83(12), 2366 (1999)

[10] M. D. Pashley, Phys. Rev. B 40, 10481 (1989)

[11] M. Biehl, M. Ahr, W. Kinzel, M. Sokolowski, T. Volkmann. unpublished, cond-mat/00008017

[12] M. E. J. Newman, G. T. Barkema. Monte Carlo Methods in Statistical Physics, Clarendon Press (Oxford, 1999)

[13] See http://theorie.physik.uni-wuerzburg.de/ ahr/AB/ for surface images and MPEG movies.

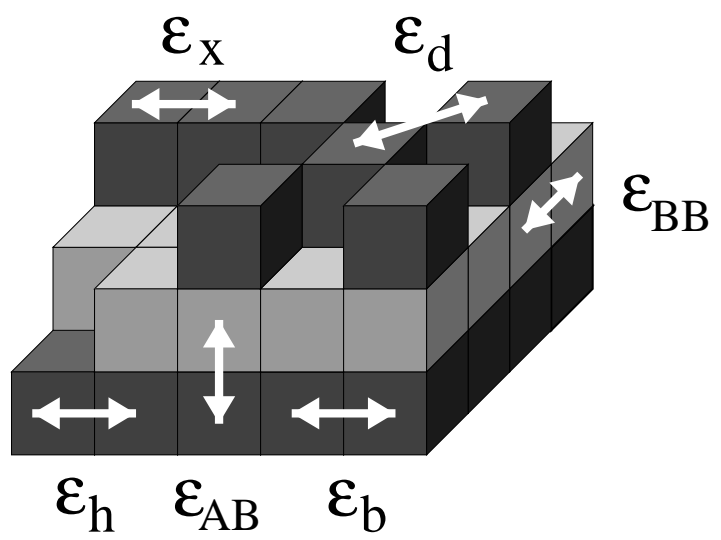

FIG. 1. Sketch of a surface showing the interactions between the particles. $A$ particles are shown as dark cubes, $B$ particles in light gray. 

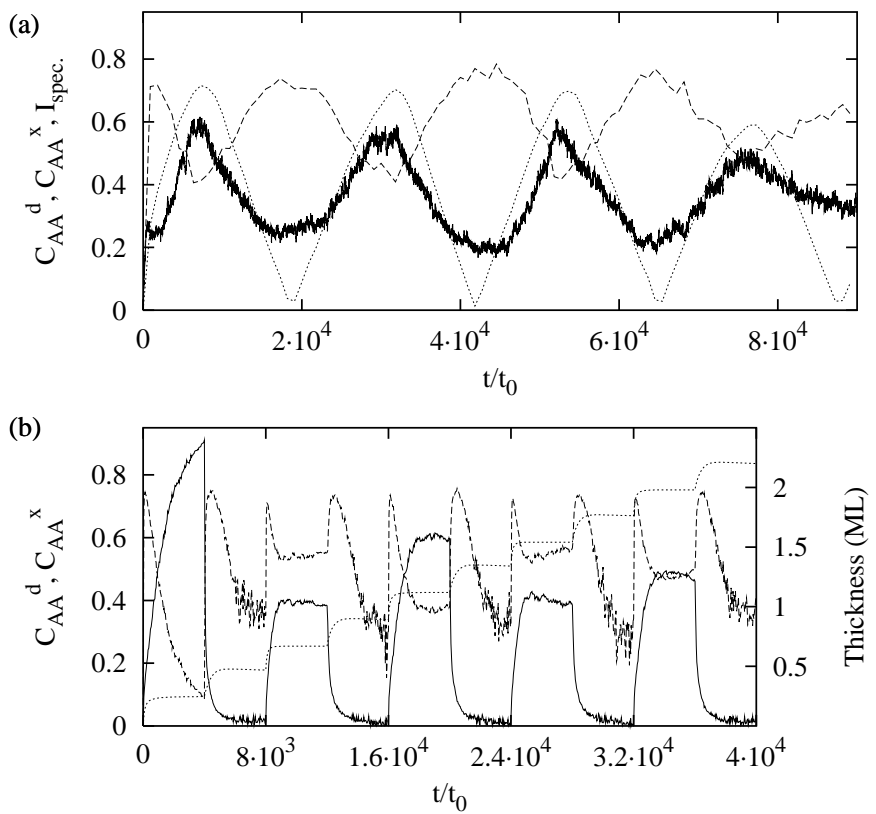

FIG. 2. Panel (a): Sublimation starting from a flat $B$-terminated surface at $T=0.5$ and a system size $N=128$. Solid lines: $C_{A A}^{d}$, dashed lines: $C_{A A}^{x}$, dotted: $I_{\text {spec. }}$ (arbitrary units). For clarity of plotting, the last two curves have been smoothed; $C_{A A}^{d}$ shows the natural fluctuations. Each oszillation period corresponds to the desorption of one ML. Panel(b): Results of ALE simulations: $C_{A A}^{d}$ (solid), $C_{A A}^{x}$ (dashed) and the thickness of the deposited film (dotted) at $T=0.3, t_{c y k l}=4 \cdot 10^{3} t_{0}$ and a particle flux of $20 \mathrm{ML} /$ cycle. The system size was $N=256$. 

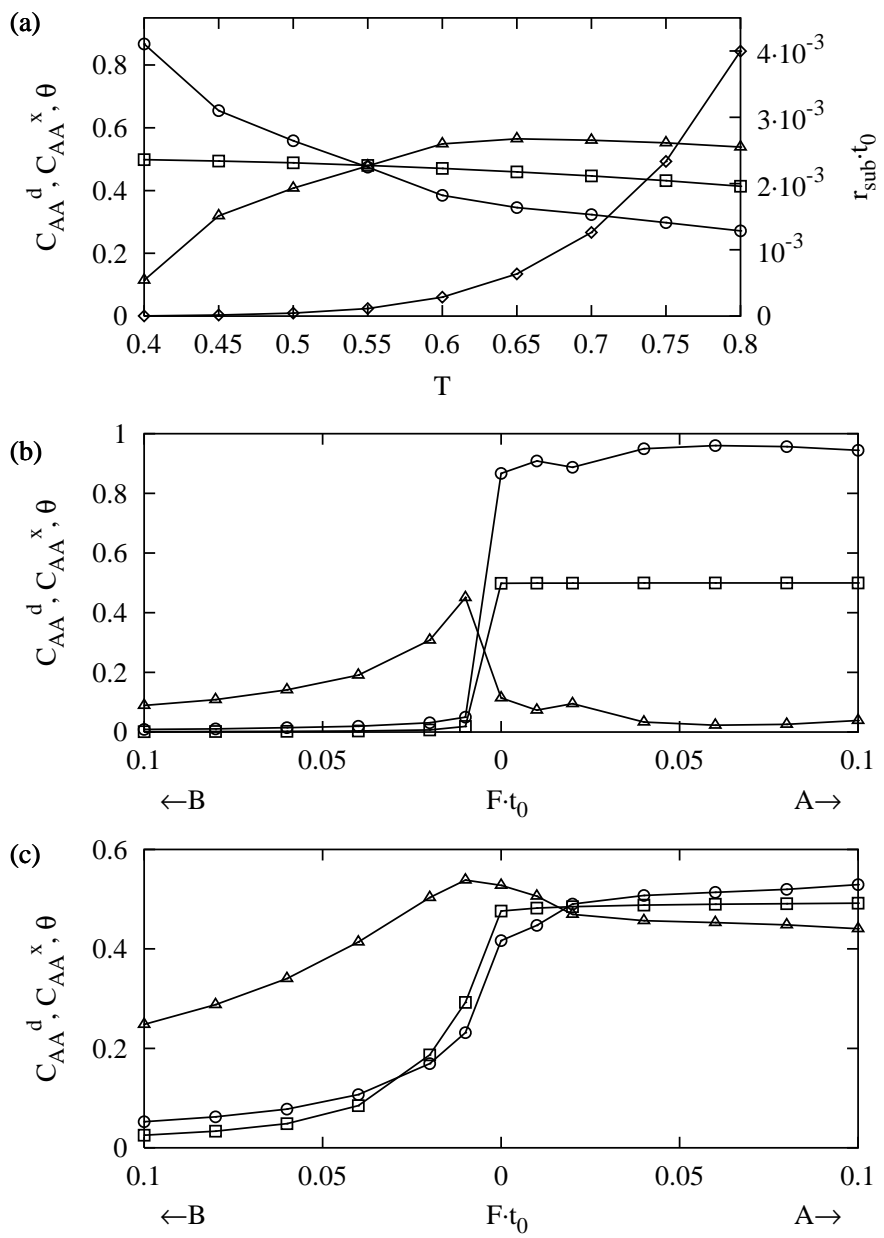

FIG. 3. The stationary state of sublimation from vicinal surfaces with terraces of $32 \sqrt{2}$ lattice constants width, oriented diagonally. (a): Temperature dependence of $\theta_{A}$ (squares), $C_{A A}^{d}$ (circles), $C_{A A}^{x}$ (triangles) and $r_{\text {sub }}$ (diamonds) under vacuum. (b), (c) The influence of an external particle flux on these quantities at $T=0.4$ (b) and $T=0.6$ (c). $A$ fluxes are shown from left to right, $B$ fluxes from right to left. Note that an $A$-flux leads to a re-entry into the $c(2 \times 2)$ reconstructed configuration at temperatures above the reordering in vacuum. Errorbars are on the order of the symbol sizes. The system size was $N=128$. Each datapoint has been obtained in a single simulation run. 

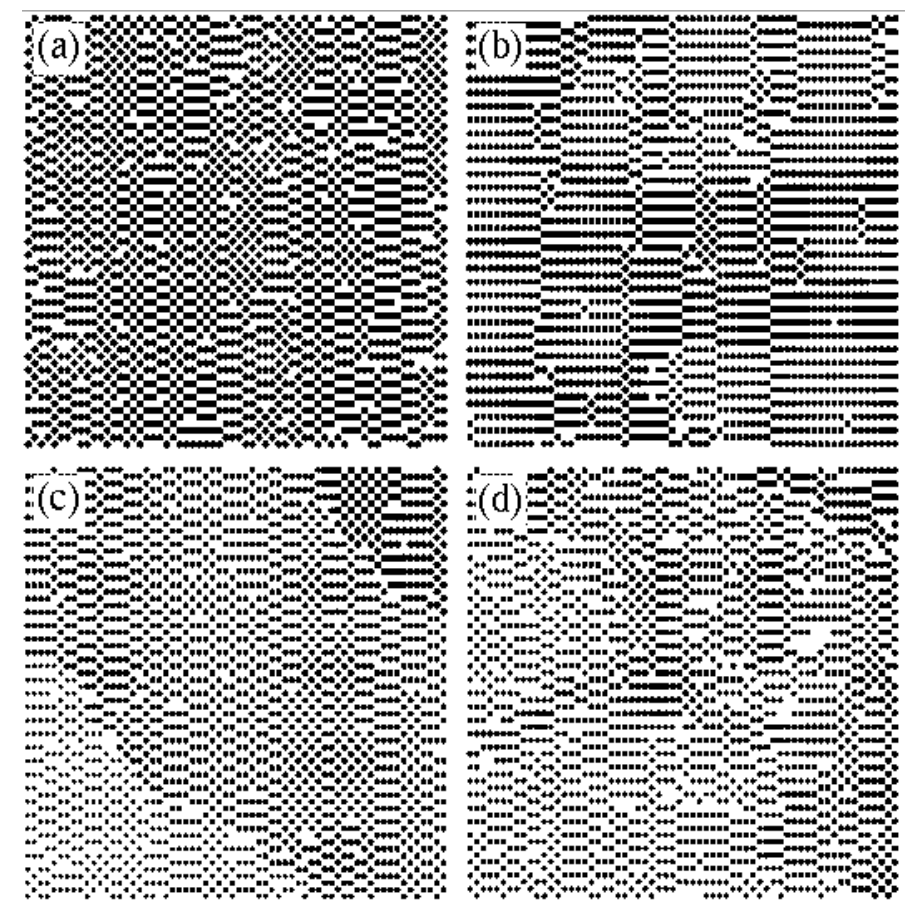

FIG. 4. Surface snapshots. The dots show colums terminated with $A$ atoms, while the $B$ atoms have been omitted for clarity. Larger dots denote higher columns. Panels (a), (b): Layer-by-layer sublimation. Panel (a) shows sections of $64 \times 64$ lattice constans of the surface in the simulation run of Figure 2 a at $t=7000 t_{0}$ (maximal $I_{\text {spec }}$ ), panel (b) at $t=1.9 \cdot 10^{4} t_{0}($ minimal $\left.I_{\text {spec }}\right)$. Panels (c), (d): Sublimation of a vicinal surface in step flow mode. The pictures show sections of surfaces at the end of simulation runs shown in Figure 3a. Panel (c): $T=0.5$ (below the transition). Panel (d): $T=0.6$ (above the transition). 